TRANSACTIONS OF THE

AMERICAN MATHEMATICAL SOCIETY

Volume 349, Number 4, April 1997, Pages 1675-1683

S 0002-9947(97)01737-6

\title{
PRIMITIVE HIGHER ORDER EMBEDDINGS OF ABELIAN SURFACES
}

\author{
TH. BAUER AND T. SZEMBERG
}

\begin{abstract}
In recent years several concepts of higher order embeddings have been studied: $k$-spannedness, $k$-very ampleness and $k$-jet ampleness. In the present note we consider primitive line bundles on abelian surfaces and give numerical criteria which allow to check whether a given ample line bundle satisfies these properties.
\end{abstract}

\section{INTRODUCTION}

The aim of this note is to study higher order embeddings of abelian surfaces defined by primitive line bundles.

In recent years several concepts of higher order embeddings have been studied, in particular $k$-spannedness, $k$-very ampleness and $k$-jet ampleness (see [7] for references on this topic). Recall that a line bundle $L$ on a smooth projective variety $X$ is called $k$-jet ample, if for any choice of distinct points $x_{1}, \ldots, x_{r}$ in $X$ and positive integers $k_{1}, \ldots, k_{r}$ with $\sum k_{i}=k+1$ the evaluation map

$$
H^{0}(X, L) \longrightarrow H^{0}\left(X, L \otimes \mathcal{O}_{X} /\left(\mathfrak{m}_{x_{1}}^{k_{1}} \otimes \ldots \otimes \mathfrak{m}_{x_{r}}^{k_{r}}\right)\right)
$$

is surjective. Further, $L$ is called $k$-very ample (resp. $k$-spanned), if for any zerodimensional subscheme $\left(Z, \mathcal{O}_{Z}\right)$ of $X$ of length $k+1$ (resp. for any curvilinear zero-dimensional subscheme $\left(Z, \mathcal{O}_{Z}\right)$ of $X$ of length $\left.k+1\right)$ the natural map

$$
H^{0}(X, L) \longrightarrow H^{0}\left(X, L \otimes \mathcal{O}_{Z}\right)
$$

is surjective. Here a subscheme is called curvilinear, if it is locally contained in a smooth curve.

There are interesting geometrical interpretations of $k$-very ampleness and $k$ spannedness in terms of secant varieties and embeddings of Hilbert schemes. On the other hand, $k$-jet ampleness has recently gained interest because of its close connection to Seshadri constants of ample line bundles.

In [4] higher order embeddings of abelian varieties defined by tensor products of ample line bundles were studied. In particular, a bound for the $k$-jet ampleness of tensor powers of ample line bundles was given:

Received by the editors December 1, 1995.

1991 Mathematics Subject Classification. Primary 14E25; Secondary 14C20.

The first author was supported by DFG contract Ba 423/7-1.

The second author was partially supported by KBN grant P03A-061-08. The final version of this paper was written during the second author's stay in Erlangen, which was made possible by Europroj support. 
Theorem ([4]). Let $X$ be an abelian variety, $L$ an ample line bundle on $X$ and $k \geq 0$. Then $m L$ is $k$-jet ample for $m \geq k+2$.

On the other extreme, one is led to consider primitive line bundles, i.e. line bundles which are not algebraically equivalent to some nontrivial power of an ample line bundle. The problem then is to give criteria for such a line bundle $L$ to define a higher order embedding in terms of suitable numerical invariants of $L$. In the case of abelian varieties certainly the type of $L$ is the obvious data. Unfortunately even for very ampleness (i.e. embeddings of order $k=1$ ) it seems to be a difficult problem to find sharp numerical criteria on abelian varieties of arbitrary dimension (see [11] for the best bounds available at present). In this note we focus on the surface case, where a complete classification can be given.

We show:

Theorem. Let $X$ be an abelian surface with Picard number 1 and let $L$ be an ample line bundle of type $(1, d), d \geq 1$. Fix a nonnegative integer $k$. Then

(a) $L$ is $k$-jet ample if and only if $d>\frac{1}{2}(k+2)^{2}$,

(b) $L$ is $k$-very ample if and only if $d \geq 2 k+3$,

(c) $L$ is $k$-spanned if and only if it is $k$-very ample.

For $k=0$ and $k=1$ these are the well-known bounds for global generation and very ampleness of primitive line bundles on abelian surfaces. It is known that in these cases the assumption on the Picard number can be weakened by the assumption that $X$ is simple (see Sect. 1). However - as will be shown in Sect. 4 - this is not possible in general. In fact the numerical criteria of the theorem may fail as soon as the Picard number is at least 2 .

Notation and Conventions. We work throughout over the field $\mathbb{C}$ of complex numbers. For a $\mathbb{Q}$-divisor $D$ we denote by $\lceil D\rceil$ its round-up and by $\lfloor D\rfloor$ its rounddown or integer part.

We would like to thank the referee for his comments on the first version of this paper.

\section{Global generation and very ampleness}

To put our investigation of higher order embeddings into perspective we briefly review the situation for global generation and very ampleness of line bundles of type $(1, d), d \geq 1$, on an abelian surface $X$.

For $d=1$ the polarized abelian surface $(X, L)$ is either the Jacobian of a smooth curve of genus 2 or the polarized product of elliptic curves - at any rate $H^{0}(X, L)$ consists of just one divisor.

If $d \geq 2$, then as a consequence of the Decomposition Theorem (see [18, Theorem 4.3.1]) the linear system $|L|$ has fixed components if and only if $X$ is a product of elliptic curves $E_{1}, E_{2}$ and $L$ is the product polarization

$$
L=\mathcal{O}_{X}\left(E_{1} \times\{0\}+d\left(\{0\} \times E_{2}\right)\right) .
$$

From now on, assume that $L$ is not of this form.

For $d=2$ the system $|L|$ is a pencil with exactly four base points. Look at [2] for more on abelian surfaces of type $(1,2)$. 
For $d \geq 3$ the linear system $|L|$ has no base points, since the base locus of $|L|$ is invariant under the action of the kernel $K(L)$ of the canonical homomorphism

$$
X \longrightarrow \operatorname{Pic}^{0}(X), \quad x \longmapsto t_{x}^{*} L-L,
$$

where $t_{x}: X \longrightarrow X, y \longmapsto x+y$, denotes the translation by $x$.

In the case $d=3$ the mapping $X \longrightarrow \mathbb{P}_{2}$ defined by $L$ is a $6: 1$-covering branched over a curve of degree 18. See [9] for a detailed investigation of a family of abelian surfaces of type $(1,3)$.

For $d=4$ the bundle $L$ cannot be very ample, since there are no abelian surfaces in $\mathbb{P}_{3}$ by the Lefschetz hyperplane theorem. Nevertheless for generic $(X, L)$ of type $(1,4)$ the map $X \longrightarrow \mathbb{P}_{3}$ is birational onto a singular octic in $\mathbb{P}_{3}$ (see [10]).

Finally, it follows from Reider's theorem that for $d \geq 5$ the line bundle $L$ is very ample if and only if there is no elliptic curve $E \subset X$ with $L C=2$ (see [18, Theorem 10.4.1]). Actually one can get much more precise information on the elliptic curves obstructing the very ampleness of $L$ (see [17]).

In sum, one has uniform bounds for global generation and very ampleness on abelian surfaces without elliptic curves:

Theorem 1.1. Let $X$ be a simple abelian surface and let $L$ be an ample line bundle of type $(1, d), d \geq 1$. Then

(a) $L$ is globally generated if and only if $d \geq 3$,

(b) $L$ is very ample if and only if $d \geq 5$.

\section{Generation of Jets}

The aim of this section is to give a sharp criterion for $k$-jet ampleness of primitive line bundles. We begin by showing:

Proposition 2.1. Let $X$ be an abelian surface with Picard number 1 , let $L$ be an ample line bundle of type $(1, d), d \geq 1$, and let $k$ be a nonnegative integer. If $d>\frac{1}{2}(k+2)^{2}$, then $L$ is $k$-jet ample.

A proof of Proposition 2.1 could be given using results of Demailly obtained by analytic methods (cf. $[13,8.12]$ ). However, we prefer to give an algebraic proof using $\mathbb{Q}$-divisors based on the

Kawamata-Viehweg Vanishing Theorem 2.2. Let $Y$ be a smooth projective surface and let $M$ be a nef and big $\mathbb{Q}$-divisor on $Y$. Then

$$
H^{i}\left(Y, \mathcal{O}_{Y}\left(K_{Y}+\lceil M\rceil\right)\right)=0 \quad \text { for } i>0 .
$$

Note that there is no normal crossing hypothesis in the surface case of KawamataViehweg vanishing thanks to Sakai's lemma (see [15, Lemma 1.1]).

Proof of Proposition 2.1. Let points $x_{1}, \ldots, x_{r}$ in $X$ and positive integers $k_{1}, \ldots, k_{r}$ with $\sum k_{i}=k+1$ be given. We will show that

$$
H^{1}\left(X, L \otimes \bigotimes_{i=1}^{r} \mathfrak{m}_{x_{i}}^{k_{i}}\right)=0 .
$$

To prove $(*)$ we are going to use $\mathbb{Q}$-divisors much as in the proof of the variant of Reider's theorem given in [15, Sect. 1]. The first step thus consists in producing an 
effective divisor with high multiplicities at the points $x_{i}$ via an elementary dimension count: For $n \geq 1$ one has by Riemann-Roch

$$
h^{0}(X, n L)=\frac{1}{2} n^{2} L^{2}=n^{2} d>\frac{1}{2}(k+2)^{2} n^{2} .
$$

On the other hand, it imposes at most

$$
\sum_{i=1}^{r}\left(\begin{array}{c}
n\left(k_{i}+1\right)+1 \\
2
\end{array}\right)=\frac{1}{2} n^{2} \sum\left(k_{i}+1\right)^{2}+O(n) \leq \frac{1}{2}(k+2)^{2} n^{2}+O(n)
$$

conditions on a divisor to have multiplicities bigger than $n\left(k_{i}+1\right)$ at the points $x_{i}$ for $1 \leq i \leq r$. This shows that for $n \gg 0$ there is a divisor $D \in|n L|$ with

$$
m_{i}=\text { def }_{\text {mult }_{x_{i}} D>n\left(k_{i}+1\right) \quad \text { for } 1 \leq i \leq r .} .
$$

Now let $f: \tilde{X} \longrightarrow X$ be the blow-up of $X$ in the points $x_{1}, \ldots, x_{r}$ with exceptional divisors $E_{1}, \ldots, E_{r}$. Consider the $\mathbb{Q}$-divisor

$$
M={ }_{\text {def }} f^{*} L-\lambda f^{*} D, \text { where } \lambda==_{\text {def }} \max \left\{\frac{k_{i}+1}{m_{i}} \mid 1 \leq i \leq r\right\} .
$$

We have $M \equiv(1-\lambda n) f^{*} L$, so $M$ is nef and big by choice of $\lambda$. Next we determine the round-up of $M$. Writing $D=\sum_{j=1}^{s} d_{j} D_{j}$ with $d_{j}>0$ and irreducible curves $D_{j}$, we get

$$
\lceil M\rceil=f^{*} L-\sum_{j=1}^{s}\left\lfloor\lambda d_{j}\right\rfloor D_{j}^{\prime}-\sum_{i=1}^{r}\left\lfloor\lambda m_{i}\right\rfloor E_{i},
$$

where $D_{j}^{\prime}$ is the proper transform of $D_{j}$. Since $\operatorname{NS}(X) \cong \mathbb{Z}$ and $L$ is primitive, any component $D_{j}$ of $D$ is algebraically equivalent to some positive multiple of $L$, hence we must have $d_{j} \leq n$. This gives the estimate $\lambda d_{j} \leq \lambda n<1$, hence $\left\lfloor\lambda d_{j}\right\rfloor=0$. Further, we have

$$
\lambda m_{i}=\max _{\ell} \frac{k_{\ell}+1}{m_{\ell}} \cdot m_{i} \geq k_{i}+1,
$$

hence $\left\lfloor\lambda m_{i}\right\rfloor \geq k_{i}+1$. Thus we find that

$$
K_{\widetilde{X}}+\lceil M\rceil=f^{*} L-\sum_{i=1}^{r}\left(\left\lfloor\lambda m_{i}\right\rfloor-1\right) E_{i}=f^{*} L-\sum_{i=1}^{r} b_{i} E_{i},
$$

where $b_{i} \geq k_{i}$. The Kawamata-Viehweg vanishing theorem then implies

$$
H^{1}\left(\widetilde{X}, f^{*} L-\sum_{i=1}^{r} b_{i} E_{i}\right)=0 .
$$

Finally, taking cohomology of the exact sequence

$$
\begin{aligned}
0 \longrightarrow \mathcal{O}_{\widetilde{X}}\left(f^{*} L-E_{j}-\sum_{i=1}^{r} k_{i} E_{i}\right) \longrightarrow \mathcal{O}_{\widetilde{X}}\left(f^{*} L-\sum_{i=1}^{r} k_{i} E_{i}\right) \\
\longrightarrow \mathcal{O}_{E_{j}}\left(f^{*} L-\sum_{i=1}^{r} k_{i} E_{i}\right) \longrightarrow 0
\end{aligned}
$$

and using induction, we see that we may replace the coefficients $b_{i}$ in the vanishing $(* *)$ by the possibly smaller numbers $k_{i}$, and the Leray spectral sequence gives the desired vanishing $(*)$ on $X$. 
Next we show that the condition $d>\frac{1}{2}(k+2)^{2}$ is also necessary for $k$-jet ampleness. This holds without the assumption on the Picard number:

Proposition 2.3. Let $L$ be an ample line bundle of type $(1, d), d \geq 1$, on an abelian surface $X$. If $L$ is $k$-jet ample, then $d>\frac{1}{2}(k+2)^{2}$.

Proof. Let $L$ be $k$-jet ample and suppose to the contrary that $d \leq \frac{1}{2}(k+2)^{2}$. Let $e \in X$ be a half-period. Taking cohomology from the exact sequence

$$
0 \longrightarrow L \otimes \mathfrak{m}_{e}^{k+1} \longrightarrow L \longrightarrow L \otimes \mathcal{O}_{X} / \mathfrak{m}_{e}^{k+1} \longrightarrow 0
$$

and using the surjectivity of $H^{0}(X, L) \longrightarrow H^{0}\left(X, L \otimes \mathcal{O}_{X} / \mathfrak{m}_{e}^{k+1}\right)$, we find that it imposes exactly $\left(\begin{array}{c}k+2 \\ 2\end{array}\right)$ conditions on a divisor in $|L|$ to have multiplicity $k+1$ in $e$, i.e.

$$
h^{0}\left(X, L \otimes \mathfrak{m}_{e}^{k+1}\right)=d-\left(\begin{array}{c}
k+2 \\
2
\end{array}\right) .
$$

Passing to a translate of $L$, we may assume that $L$ is symmetric, i.e. $\iota^{*} L \cong L$, where $\iota: X \longrightarrow X$ is the $(-1)$-involution. We will derive a contradiction with $(*)$ considering the number of conditions imposed on even sections of $L$. For this recall that the space of global sections of $L$ admits a decomposition

$$
H^{0}(X, L)=H^{0}(X, L)^{+} \oplus H^{0}(X, L)^{-}
$$

into even and odd sections. After possibly replacing $L$ by another symmetric translate the respective dimensions are given by

$$
h^{0}(X, L)^{ \pm}= \begin{cases}\frac{d \pm 1}{2} & \text { if } d \text { is odd } \\ \frac{d \pm 2}{2} & \text { if } d \text { is even }\end{cases}
$$

(see [18, Corollary 4.6.6]).

We first consider the case that $k$ is even. Note that the multiplicity of an even section in an odd half-period is always odd. So if we choose the half-period $e$ to be odd, then the number of conditions on an even section imposed by vanishing in $e$ to the order $k+1$ is at most

$$
2+4+\cdots+k=2 \cdot\left(\begin{array}{c}
\frac{k}{2}+1 \\
2
\end{array}\right) .
$$

Thus we have the estimate

$$
h^{0}\left(X, L \otimes \mathfrak{m}_{e}^{k+1}\right)^{+} \geq h^{0}(X, L)^{+}-2 \cdot\left(\begin{array}{c}
\frac{k}{2}+1 \\
2
\end{array}\right)=\left\lceil\frac{d+1}{2}\right\rceil-\frac{1}{4}\left(k^{2}+2 k\right) .
$$

Using $(*)$ we find the inequality

$$
0 \leq h^{0}\left(X, L \otimes \mathfrak{m}_{e}^{k+1}\right)-h^{0}\left(X, L \otimes \mathfrak{m}_{e}^{k+1}\right)^{+} \leq d-\left\lceil\frac{d+1}{2}\right\rceil-\frac{(k+2)^{2}}{4},
$$

which gives a contradiction with the assumption $d \leq \frac{1}{2}(k+2)^{2}$. This completes the proof in case $k$ is even. For $k$ odd a similar argument works considering an even half-period. 


\section{3. $k$-VERY AMPLE AND $k$-SPANNED LINE BUNDLES}

In this section we prove an analogue of Theorem 1.1 for $k$-very ampleness. We begin by recalling the numerical criterion for $k$-very ampleness given by Beltrametti and Sommese.

Theorem $3.1([5])$. Let $L$ be a nef line bundle on a smooth projective surface $Y$ with $L^{2} \geq 4 k+5, k \geq 0$. Then the following conditions are equivalent:

(i) The adjoint bundle $K_{Y}+L$ is not $k$-very ample.

(ii) There is an effective divisor $D$ on $Y$ such that $L-2 D$ is $\mathbb{Q}$-effective, $D$ contains some 0-dimensional subscheme where the $k$-very ampleness fails, and

$$
L D-(k+1) \leq D^{2}<\frac{L D}{2}<k+1 .
$$

As an easy consequence we get the following criterion for $k$-very ampleness:

Proposition 3.2. Let $X$ be an abelian surface with Picard number 1 , let $L$ be an ample line bundle of type $(1, d), d \geq 1$, and let $k$ be a nonnegative integer. If $d \geq 2 k+3$, then $L$ is $k$-very ample.

For the proof of the proposition note first that $L^{2}=2 d \geq 4 k+6$ by assumption. Further, if $D$ is any effective divisor on $X$, then we have $D \equiv$ alg $p L$ for some $p \geq 1$, so

$$
L D=p L^{2} \geq 4 k+6,
$$

which shows that a divisor as in condition (ii) of Theorem 3.1 cannot exist.

The harder part is to show that the bound $2 k+3$ is sharp. Actually this is already the sharp bound for $k$-spannedness:

Proposition 3.3. Let $L$ be an ample line bundle of type $(1, d), d \geq 1$, on an abelian surface $X$. If $L$ is $k$-spanned, then $d \geq 2 k+3$.

Proof. The analogous statement for $k$-very ampleness follows from [1]. However, the argument extends to the case of $k$-spannedness. We briefly sketch it to make this clear.

Theorem 1.1 implies that the proposition holds for $k=0$ and $k=1$. So in particular $L$ may be assumed to be very ample. Suppose then that $L$ is $k$-spanned and $d \leq 2 k+2$. Let

$$
\varphi: A \longrightarrow \mathbb{P}\left(H^{0}(L)\right)=\mathbb{P}_{d-1}
$$

be the embedding given by $L$ and let $C \in|L|$ be a smooth curve. The exact sequence

$$
0 \longrightarrow \mathcal{O}_{X} \longrightarrow L \longrightarrow L \otimes \mathcal{O}_{C} \longrightarrow 0
$$

and Riemann-Roch imply that $C$ is not projectively normal in $\mathbb{P}_{d-1}$. Let $D \in$ $\mathbb{P}\left(H^{0}\left(L \otimes \mathcal{O}_{C}\right)\right)$ be the image of $C$ under the embedding defined by the complete linear system $\left|L \otimes \mathcal{O}_{C}\right|$. The restriction map $\alpha: H^{0}(L) \longrightarrow H^{0}\left(L \otimes \mathcal{O}_{C}\right)$ induces the projection

$$
\alpha^{*}: \mathbb{P}\left(H^{0}\left(L \otimes \mathcal{O}_{C}\right)\right) \longrightarrow \mathbb{P}\left(H^{0}(L)\right)
$$

from the line $Q=\mathbb{P}(\operatorname{coker} \alpha)$. The restriction of this projection to $D$ gives an isomorphism $D \cong C$.

Now consider the $k$-th secant variety $\operatorname{Sec}^{k}(D)$ of $D$. By assumption $d-1 \leq 2 k+1$, so Zak's formula (see [21]) implies

$$
\operatorname{dim} \operatorname{Sec}^{k}(D)=\min \{d, 2 k+1\} \geq d-1 ;
$$


hence there is a point $P \in \operatorname{Sec}^{k}(D) \cap Q$. This means that there is a 0 -dimensional subscheme $Z_{D}$ of $D$ of length $k+1$ such that

$$
P \in \mathbb{P}\left(H^{0}\left(L \otimes \mathcal{O}_{C} \otimes \mathcal{I}_{Z_{D}}\right)\right)
$$

Then $Z=\alpha^{*}\left(Z_{D}\right)$ is a curvilinear subscheme of length $k+1$ on $A$. Since $L \otimes \mathcal{O}_{C}$ is $k$-spanned and $P$ lies in the center of the projection $\alpha^{*}$, we get

$$
\begin{aligned}
d-(k+1)-1 & =\operatorname{dim} \mathbb{P}\left(H^{0}\left(L \otimes \mathcal{I}_{Z}\right)\right)=\operatorname{dim} \alpha^{*}\left(\mathbb{P}\left(H^{0}\left(L \otimes \mathcal{O}_{C} \otimes \mathcal{I}_{Z_{D}}\right)\right)\right) \\
& <\operatorname{dim} \mathbb{P}\left(H^{0}\left(L \otimes \mathcal{O}_{C} \otimes \mathcal{I}_{Z_{D}}\right)\right)=d-(k+1)-1
\end{aligned}
$$

a contradiction.

Remark 3.4. We would like to point out here that Theorem 3.1 also leads to a bound for $k$-jet ampleness. First recall the following relation between $k$-very ampleness and $k$-jet ampleness (see [6, Proposition 2.1]): If $L$ is $k^{\prime}$-very ample, then $L$ is $k$-jet ample, where $k$ is the biggest integer such that

$$
\left(\begin{array}{c}
k+2 \\
2
\end{array}\right) \leq k^{\prime}+1
$$

Suppose now that we want to show the $k$-jet ampleness of a line bundle of type $(1, d)$ on an abelian surface $X$ for suitable $d \geq 1$. According to $(*)$ it is enough to show $k^{\prime}$-very ampleness, where $k^{\prime}=\left(\begin{array}{c}k+2 \\ 2\end{array}\right)-1$. Assuming $\rho(X)=1$ and using Theorem 3.1, this will follow as soon as $L^{2} \geq 4 k^{\prime}+5$, which is equivalent to

$$
d \geq k^{2}+3 k+3 \text {. }
$$

So the bound one gets in this way is also quadratic in $k$, but it is considerably bigger than the optimal bound $d>\frac{1}{2}(k+2)^{2}$ that we gave in Sect. 2 .

\section{Surfaces with higher Picard number}

One might be tempted to hope that uniform numerical criteria for $k$-jet ampleness and $k$-very ampleness could be given for simple abelian surfaces, rather than for abelian surfaces with Picard number 1 - just as it is the case for global generation and very ampleness in Theorem 1.1. However, for a line bundle of type $(1, d)$ the implications

(i) if $d>\frac{1}{2}(k+2)^{2}$, then $L$ is $k$-jet ample,

(ii) if $d \geq 2 k+3$, then $L$ is $k$-very ample,

may fail as soon as the Picard number gets bigger than 1 , even for simple abelian surfaces. We will illustrate this by considering a two-dimensional family of simple abelian surfaces, where this happens.

4.1. In our discussion we are going to consider Seshadri constants. Recall that for a nef line bundle $L$ on a smooth projective variety $X$ the Seshadri constant of $L$ at a point $x \in X$ is by definition the real number

$$
\varepsilon(L, x)=\inf _{C \ni x} \frac{L C}{\operatorname{mult}_{x}(C)},
$$

where the infimum is taken over all irreducible curves $C \subset X$ containing $x$. The Seshadri constant is to be thought of as measuring the local positivity of $L$ at $x$. It is bounded from above by $\varepsilon(L, x) \leq \sqrt{L^{d}}$, where $d=\operatorname{dim} X$ (see [14, Remark 1.8]). There is an interesting connection between Seshadri constants and the generation 
of jets: Denote by $s(L, x)$ the maximal number $s$ such that $L$ generates $s$-jets at $x$, i.e. such that

$$
H^{0}(X, L) \longrightarrow H^{0}\left(X, L \otimes \mathcal{O}_{X} / \mathfrak{m}_{x}^{s+1}\right)
$$

is onto. If $L$ is ample, then

$$
\varepsilon(L, x)=\limsup _{n \longrightarrow \infty} \frac{s(n L, x)}{n}
$$

(see [12, Theorem 6.4]).

Finally, note that if $X$ is an abelian variety, then by homogeneity $\varepsilon(L)=\varepsilon(L, x)$ is actually independent of the point $x$.

4.2. Now we turn to the examples. Let $X$ be a principally polarized abelian surface with endomorphism ring $\mathbb{Z}[\sqrt{7}]$. (There is a two-dimensional family of such surfaces, cf. [8, Sect. 2].) The abelian surface $X$ does not contain elliptic curves and its Néron-Severi group is generated by line bundles $L_{0}$ and $L_{1}$ of types $(1,1)$ (resp. $(1,9))$ with $L_{0} L_{1}=8$ (cf. [3, Sect. 3]).

We consider the line bundles

$$
M_{n}=_{\text {def }} n L_{0}-L_{1} \quad(n \geq 1)
$$

on $X$. For $n \gg 0$ these bundles are certainly ample and primitive. $M_{n}$ is of type $\left(1, d_{n}\right)$ with $d_{n}=n^{2}-8 n+9$. Put

$$
k_{n}=_{\text {def }}\left\lfloor\sqrt{2 n^{2}-16 n+18}\right\rfloor-3 .
$$

Then we have $d_{n}>\frac{1}{2}\left(k_{n}+1\right)^{2}$, so if we assume the criterion (i) to hold on $X$, then $M_{n}$ had to be $k_{n}$-jet ample. In particular it should generate $k_{n}$-jets at any point. Since we have an inclusion

$$
H^{0}\left(X, M_{n}\right) \hookrightarrow H^{0}\left(X, n L_{0}\right)
$$

we get $s\left(n L_{0}\right) \geq s\left(M_{n}\right) \geq k_{n}$, which using $\varepsilon\left(L_{0}\right) \leq \sqrt{L_{0}^{2}}=\sqrt{2}$ implies that the Seshadri constant of $L_{0}$ is maximal:

$$
\varepsilon\left(L_{0}\right)=\limsup _{n \longrightarrow \infty} \frac{s\left(n L_{0}\right)}{n}=\sqrt{2} .
$$

On the other hand, one can use Steffens' argument [20] to bound $\varepsilon\left(L_{0}\right)$ from below: There is a curve $C$ in $\left|4 L_{0}\right|$ with mult $_{0}(C)=6$, namely the image of the unique divisor $\Theta \in\left|L_{0}\right|$ under the endomorphism $2_{X}: X \longrightarrow X$ given as multiplication by 2 , so we have

$$
\varepsilon\left(L_{0}\right) \leq \frac{L_{0} C}{\operatorname{mult}_{0}(C)}=\frac{4}{3},
$$

a contradiction with $(*)$.

Next assume that criterion (ii) holds on $X$. This would imply that $M_{n}$ is $k_{n}^{\prime}$-very ample, where

$$
k_{n}^{\prime}={ }_{\text {def }}\left\lfloor\frac{n^{2}-8 n+6}{2}\right\rfloor .
$$

On the other hand, for a divisor $D \in\left|L_{1}\right|$ we have

$$
M_{n} D=8 n-18,
$$

so for $n \gg 0$ we certainly have $M_{n} D<k_{n}^{\prime}$, which is clearly impossible for a $k_{n}^{\prime}$-very ample line bundle. 


\section{REFERENCES}

[1] Ballico, E., Sommese, A.J.: Projective surfaces with $k$-very ample line bundles of degree $\leq 4 k+4$. Nagoya Math. J. 136, 57-79 (1994) MR 96d:14005

[2] Barth, W.: Abelian Surfaces with (1,2)-Polarisation. Algebraic Geometry (Sendai, 1985), Advanced Studies in Pure Math., vol. 10, North-Holland, Amsterdam, 1987, pp. 41-84. MR 89i: 14038

[3] Bauer, Th.: Quartic surfaces with 16 skew conics. J. Reine Angew. Math. 464, 207-217 (1995) MR 96j; 14024

[4] Bauer, Th., Szemberg, T.: Higher order embeddings of abelian varieties. Math. Z. (to appear)

[5] Beltrametti, M.C., Sommese, A.J.: Zero cycles and $k$-th order embeddings of smooth projective surfaces. Projective Surfaces and Their Classification, Symp. Math., INDAM, vol. 32, Academic Press, New York, 1988, pp. 33-48. MR 95d:14005

[6] Beltrametti, M.C., Sommese, A. J.: On $k$-jet ampleness. Complex Analysis and Geometry, edited by V. Ancona and A. Silva, Plenum Press, New York, 1993, pp. 355-376. MR 94g:14006

[7] Beltrametti, M.C., Sommese, A.J.: The adjunction theory of complex projective varieties. De Gruyter Expositions in Math. 16, de Gruyter, Berlin, 1995. MR 96f:14004

[8] Birkenhake, Ch.: Tensor products of ample line bundles on abelian varieties. Manuscripta Math. 84, 21-28 (1994) MR 95i:14040

[9] Birkenhake, Ch., Lange, H.: A family of abelian surfaces and curves of genus four. Manuscripta Math. 85, 393-407 (1994) MR 95k:14064

[10] Birkenhake, Ch., Lange, H., van Straten, D.: Abelian surfaces of type (1,4). Math. Ann. 285, 625-646 (1989) MR 91b:14042

[11] Debarre, O., Hulek, K., Spandaw, J.: Very ample linear systems on abelian varieties. Math. Ann. 300, 181-202 (1994) MR 95k:14065

[12] Demailly, J.-P.: Singular Hermitian metrics on positive line bundles. Complex Algebraic Varieties (Bayreuth, 1990), Lect. Notes Math. 1507, Springer-Verlag, 1992, pp. 87-104. MR 93g:32044

[13] Demailly, J.-P.: $L^{2}$ vanishing theorems for positive line bundles and adjunction theory. To appear.

[14] Ein, L., Küchle, O., Lazarsfeld, R.: Local positivity of ample line bundles. J. Diff. Geom. 42, 193-219 (1995) MR 96m:14007

[15] Ein, L., Lazarsfeld, R.: Global generation of pluricanonical and adjoint linear series on smooth projective threefolds. J. Amer. Math. Soc. 6, 875-903 (1993) MR 94c:14016

[16] Ein, L., Lazarsfeld, R.: Seshadri constants on smooth surfaces. Astérisque 218, 177-186 (1993) MR 95f: 14031

[17] Hulek, K., Lange, H.: Examples of abelian surfaces in $\mathbb{P}_{4}$. J. Reine Angew. Math. 363, 200-216 (1985) MR 87g:14038

[18] Lange, H., Birkenhake, Ch.: Complex Abelian Varieties. Springer-Verlag, 1992. MR 94j:14001

[19] Lazarsfeld, R.: Lectures on linear series. Park City / IAS Mathematics series vol. 3, 1-56 (1993)

[20] Steffens, A.: Remarks on Seshadri constants. Preprint

[21] Zak, F.: Linear systems of hyperplane sections on varieties of low codimension. Functional Anal. Appl. 19, 165-173 (1985) MR 87d:14040 (of Russian original)

Mathematisches Institut, Universität Erlangen-Nürnberg, Bismarckstrasse $1 \frac{1}{2}$, D91054 Erlangen, Germany

E-mail address: bauerth@mi.uni-erlangen.de

Instytut Matematyki, Uniwersytet Jagielloński, Reymonta 4, PL-30-059 Kraków, POLAND

E-mail address: szemberg@im.uj.edu.pl 